\title{
A Geomagnetic Reference Field for Spain at 1990
}

\author{
J. M. Torta ${ }^{1}$, A. GARcía ${ }^{2}$, and A. DE SANTIS ${ }^{3}$ \\ ${ }^{1}$ Observatori de l'Ebre, C.S.I.C., Roquetes (Tarragona), Spain \\ ${ }^{2}$ Museo Nacional de Ciencias Naturales, C.S.I.C., Madrid, Spain \\ ${ }^{3}$ Istituto Nazionale di Geofisica, Roma, Italy
}

(Received October 5, 1992; Revised April 19, 1993)

\begin{abstract}
A Spanish Geomagnetic Reference Field for epoch 1990 has been produced using the technique of spherical cap harmonic analysis (SCHA). The data set on which the model is based includes both Magsat and ground (observatory and repeat-station) three-component data. The field data have been updated to 1990 by means of a spherical cap harmonic model of secular variation for a spherical cap of half-angle $16^{\circ}$ for the time interval 1970-1989.5. Due to the poor distribution of the ground data (almost entirely restricted to the mainland areas) we have confined the analysis to a smaller cap $\left(8^{\circ}\right)$. Before applying SCHA, field values from the IGRF 1985, evaluated at 1990, were removed from the data and only the residuals were analyzed. We found that the removal of a trend from the satellite residuals improved the fit of the resulting model to the ground data; we also found some difficulties using SCHA to extrapolate Magsat measurements to the ground. Appropriate solutions to this problem are presented. Charts of the elements of the 1990 Spanish Geomagnetic Reference Field (after the addition of the field values computed using the IGRF model) are shown, along with the secular variation maps for that epoch as deduced from the secular variation model.
\end{abstract}

\section{Introduction}

Spherical cap harmonic (SCH) analysis (HAINES, 1985a) is suitable for regional modelling of magnetic fields due to internal sources since it is based on a spherical harmonic expansion of the magnetic potential, as in global modelling, but it uses more generalized Legendre functions with harmonic degree $n_{k}(m)$, which can be non-integer (HAINES, 1985a; HAINES and NEWITT, 1986):

$$
V=a \sum_{k=0}^{K} \sum_{m=0}^{k}\left(\frac{a}{r}\right)^{n_{k}(m)+1} P_{n_{k}(m)}^{m}(\cos \theta) \sum_{p=0}^{P}\left\{G_{k, p}^{m} \cos (m \phi)+H_{k, p}^{m} \sin (m \phi)\right\}\left(t-t_{0}\right)^{p}
$$

where $r$ is the radial distance, $\theta$ is the colatitude and $\phi$ the longitude; $a$ is the reference radius; $t$ is a time or epoch, with $t_{0}$ a convenient zero time. $P_{n_{k}(m)}^{m}$ are the associated Legendre functions of the first kind with integer order $m$ and non-integer degree $n_{k}(m)$. The values of $n_{k}(m)$ are determined as the roots of $P_{n_{k}(m)}^{m}$ or $\mathrm{d} P_{n_{k}(m)}^{m} / \mathrm{d} \theta$ at the boundary of the spherical cap. $k$ is an integer index selected to arrange, in increasing order, the different roots $n_{k}$ for a given $m$, and $K$ is the maximum index. The constants $G_{k, q}{ }^{m}, H_{k, q}{ }^{m}$ are known as the spherical cap harmonic coefficients. The parameters $p$ and $P$ are the order and maximum order of the polynomial in $t$, respectively.

The potential is a solution of Laplace's equation and the basis functions that result from applying the two different sets of boundary conditions above mentioned form two distinct sețs within which each function is orthogonal to all the other basis functions. However, functions belonging to different sets are not orthogonal, but the use of functions from both sets ensures rapid uniform convergence of expansions of all derivatives of the potential (HAINES, 1990). A practical advantage of SCHA is that it is really a regional technique, in that (a) it uses the regional data alone, without requiring data outside the area of 
study; (b) it describes shorter wavelengths than do global models, like the IGRF (e.g. BARRACLOUGH, 1987).

The importance of a three-dimensional model of the regional geomagnetic field is evident: it can be used to describe the most significant characteristics of the intermediate and long-wavelength parts of the field in the region of interest (i.e., those parts of the field which are believed to originate in the Earth's fluid core plus the field due to broad-scale crustal anomalies), because a SCH model can depict features of shorter wavelength that can be described by a global model (NEWITT and HAINES, 1989). The model can also be used as a reference field when considering much shorter wavelengths in local studies. Usually the data are available at different epochs, while the geomagnetic field changes slowly with time, the so-called secular variation (SV). To have a "snapshot" of the geomagnetic field we need a SV model to reduce all the data to the same epoch. For the above reasons we define a reference field as the combination of a SV model and a model at a fixed epoch that accounts for both the field from the core and broad-scale crustal anomalies. Since main field is the standard expression for the field from the core, hereafter we will call this combination of main field plus regional anomaly field simply as fixed field, to distinguish it from the SV field. Consequently this study will describe (a) the SV model used to reduce the data, (b) the fixed field data sets and analysis, and finally, (c) the complete reference field.

\section{Secular Variation Field Analyses}

The advantage of a SCH SV model to update data within the temporal range of validity of the model itself, has already been pointed out by HAINES (1985b), HAINES and NEWITT (1986) and NEWITT and HAINES (1989). Since the derivative of a polynomial is again a polynomial, formula (1) holds not only for the fixed field but also for any of its time derivatives, such as secular variation (HAINES, 1985b). Therefore, for convenience, substituting $V, p$ and $P$ by $\dot{V}, q$ and $Q$ respectively in (1), and $G$ 's and $H$ 's by $g$ 's and $h$ 's, we can write:

$$
\dot{V}=a \sum_{k=0}^{K} \sum_{m=0}^{k}\left(\frac{a}{r}\right)^{n_{k}(m)+1} P_{n_{k}(m)}^{m}(\cos \theta) \sum_{q=0}^{Q}\left\{g_{k, q}^{m} \cos (m \phi)+h_{k, q}^{m} \sin (m \phi)\right\}\left(t-t_{0}\right)^{q}
$$

where, for $p>0, q=p-1, Q=P-1$ and $g_{k, q}{ }^{m}=p G_{k, p}{ }^{m}=(q+1) G_{k, q+1}{ }^{m}$; and similar relations for the $h^{\text {'s }}$ and $H$ 's. Hence the secular variation of the field (derived as the negative gradient of the potential $\dot{V}$ ) can be expressed as:

$$
\dot{\vec{B}}(\vec{s}, t)=-\nabla \dot{V}=\sum_{q=0}^{Q} \vec{b}_{q}(\vec{s})\left(t-t_{0}\right)^{q}
$$

where $\vec{s}$ denotes the spatial dependence and $\left(t-t_{0}\right)$ the time dependence. $\vec{b}_{q}(\vec{s})$ has north, east and vertically downward ( $b_{q N}, b_{q E}$ and $b_{q V}$, respectively) components:

$$
\begin{aligned}
& b_{q N}=\sum_{k=1}^{K} \sum_{m=0}^{k}\left(\frac{a}{r}\right)^{n_{k}(m)+2} \frac{\mathrm{d} P_{n_{k}(m)}^{m}(\cos \theta)}{\mathrm{d} \theta}\left\{g_{k, q}^{m} \cos (m \phi)+h_{k, q}^{m} \sin (m \phi)\right\}, \\
& b_{q E}=\sum_{k=1}^{K} \sum_{m=1}^{k}\left(\frac{a}{r}\right)^{n_{k}(m)+2} \frac{m P_{n_{k}(m)}^{m}(\cos \theta)}{\sin \theta}\left\{g_{k, q}^{m} \sin (m \phi)-h_{k, q}^{m} \cos (m \phi)\right\}, \\
& b_{q V}=-\sum_{k=0}^{K} \sum_{m=0}^{k}\left[n_{k}(m)+1\right]\left(\frac{a}{r}\right)^{n_{k}(m)+2} P_{n_{k}(m)}^{m}(\cos \theta)\left\{g_{k, q}^{m} \cos (m \phi)+h_{k, q}^{m} \sin (m \phi)\right\} .
\end{aligned}
$$


The field may be determined by integration:

$$
\vec{B}(\vec{s}, t)=\vec{B}\left(\vec{s}, t_{0}\right)+\sum_{q=0}^{Q} \frac{1}{q+1} \vec{b}_{q}(\vec{s})\left(t-t_{0}\right)^{q+1}
$$

where the integration constant is the field at the epoch $t_{0}$, which is independently defined in terms of its potential (as will be shown in the next two sections). In this way, if we have a SV model like (3) it is possible, with the same set of coefficients, to update field measurements from one epoch to another by using the integrated model (7) (without needing the integration constant if only a knowledge of the changes in the magnetic field is needed; HAINES, 1985b). Here we followed the "derivative-fit" method according to HAINES' (1985b) terminology. This method consists of numerically differentiating the main-field data (in practice with first central differences divided by the differencing interval) and then fitting a SV model to them. The main advantage of this procedure is to avoid the possible crustal contamination in nearsurface main-field observations which can bias the determination of the temporal coefficients.

Recent work (GARCÍA et al., 1991; TORTA et al., 1992) has shown two new procedures to obtain a model of the SV (for Spain) using spherical cap harmonic analysis (SCHA). The data, observatory annual means and measurements from repeat stations, were restricted to a cap of half-angle $16^{\circ}$, centered at $34^{\circ} \mathrm{N}$, $7^{\circ} \mathrm{W}$. The reason for considering a $16^{\circ}$ half-angle cap was to include not only the Iberian Peninsula but also the Canary Islands. To achieve a better fit to the data than was obtained by straight-forward application of SCHA, it was applied to the horizontal $(X$ and $Y$ ) and vertical $(Z)$ SV components separately (GARCÍA et al., 1991). This improved fit was obtained at the expense of a partial violation of Laplace's equation (as the SV components were not derivable from the same magnetic potential).

Later, TORTA et al. (1992) found that a problem in applying SCHA was the lack of long wavelength harmonic functions in a SCH SV model defined for a relatively small cap. This is due to the fact that the lowest values of the harmonic degree $n_{k}(m)$ over a small cap region are not as low as those typical of the SV field. Notice also that the wavelength associated with Legendre functions of degree $n_{k}(m)$ is the ratio between the terrestrial circumference and $n_{k}(m)$ (BULLARD, 1967; HAINES, 1988); and the minimum value of $n_{k}(m)$ decreases as the cap half-angle increases (excluding $k$ equal to zero, because the term corresponding to $k=0$ in the potential expansion does not really contribute to the wavelength computation; it only represents the mean value of the vertical component expansion at the Earth's surface). This problem did not arise in previous applications (HAINES, 1985b; NEWITT and HAINES, 1989) because a larger cap was considered. In order to remove this inconvenience, without making the compromise of GARCÍA et al. (1991), the SCHA was applied over a larger cap of half-angle $45^{\circ}$ with the same spherical cap pole (using the original data set) (TORTA et al., 1992). They demonstrated that the resulting model had a suitable harmonic content, coherent with the actual SV field that was being modelled. The choice of a $45^{\circ}$ cap model followed from considerations on both standard error and spectral content (this spherical cap was the best compromise for including both proper long and short wavelengths).

Since the intention was to produce a reference field model for 1990 , it was thought sensible to revise the previous SV model, which was originally based on data from the time interval 1970-1988 (TORTA et al., 1992). This revision includes newly available observatory annual mean values (GOLOVKOV and DIMANT, 1990) and repeat station measurements (Instituto Geográfico Nacional, Spain, personal communication). The new data comprise 7 new three-component observatory annual means (Coimbra, 1989; San Pablo, Almería, and Santa Cruz de Tenerife, 1988 and 1989) and 58 repeat station data, including some values from the Italian magnetic network (Istituto Nazionale di Geofisica, Italy, personal communication). The analysis of the first differences was again carried out as described in TORTA et al. (1992) with maximum spatial index $K=4$, and temporal degree $Q=2$, over a $45^{\circ}$ cap, with data limited to the $16^{\circ}$ cap, from 1970 onwards. The model was referenced to 1990.0 ; the previous one was referenced to 1987.5. After the elimination of 93 (out of 664) three-component data for which at least one component differed by more than twice the standard deviation of a preliminary fit, the new SV analysis showed a standard error of 9.45 
Table 1. Statistically significant coefficients of the SCH secular variation model, relative to 1990.0 .

\begin{tabular}{rrrrrrrrr}
\hline$k$ & $m$ & $n_{k}(m)$ & $g_{k, 0}{ }^{m}$ & $h_{k, 0}{ }^{m}$ & $g_{k, 1}{ }^{m}$ & $h_{k, 1}{ }^{m}$ & $g_{k, 2}{ }^{m}$ & $h_{k, 2}{ }^{m}$ \\
\hline 0 & 0 & .0000 & .0 & & .0 & & .1 & \\
1 & 0 & 2.5479 & 35.5 & & .0 & & -.1 & \\
1 & 1 & 2.0000 & .0 & -30.3 & .0 & 2.4 & .1 & .1 \\
2 & 0 & 4.4053 & -22.5 & & -.6 & & .0 & \\
2 & 1 & 4.4053 & 15.4 & .0 & .0 & .0 & .0 & .0 \\
2 & 2 & 3.6332 & -5.3 & -7.8 & .0 & .0 & .0 & .0 \\
3 & 0 & 6.5222 & .0 & & -.2 & & .0 & \\
3 & 1 & 6.3339 & -4.0 & .0 & .0 & .0 & .0 & .0 \\
4 & 2 & 8.1373 & .8 & .0 & .0 & .0 & .0 & .0 \\
\hline
\end{tabular}

The second subscript of the coefficients refers to the order $q$ of the temporal polynomial.

$\mathrm{nT} /$ year and 15 coefficients statistically significant at the $F$-level 4 , results that are similar to those of TORTA et al. (1992). The coefficients of the new SV model are listed in Table 1, where their second subscript refers directly to the order $q$ of the temporal expansion of (2).

\section{Fixed Field Data Sets}

For the analysis of the fixed field two data sets were used: (a) Magsat satellite and (b) ground data. The first set comprised 5615 three-component measurements made at an altitude of about $400 \mathrm{~km}$ during quiet periods. Because of the non-uniform distribution of the ground data, it was decided to use a smaller cap, excluding the Canary Islands but improving the areal coverage of the data. The new cap has a halfangle of $8^{\circ}$ with a new pole at $41^{\circ} \mathrm{N}, 3^{\circ} \mathrm{W}$, and is contained within the $45^{\circ}$ cap of the $\mathrm{SV}$ analysis. The selection of data for magnetically quiet times was made following the procedure described by TORTA $e t$ al. (1991) when modelling the anomaly field (using measurements made at 5 -second intervals, or $37 \mathrm{~km}$ along the satellite track when $K p<2+$ ).

The second set of data comprised the most recent available observatory annual means and survey data

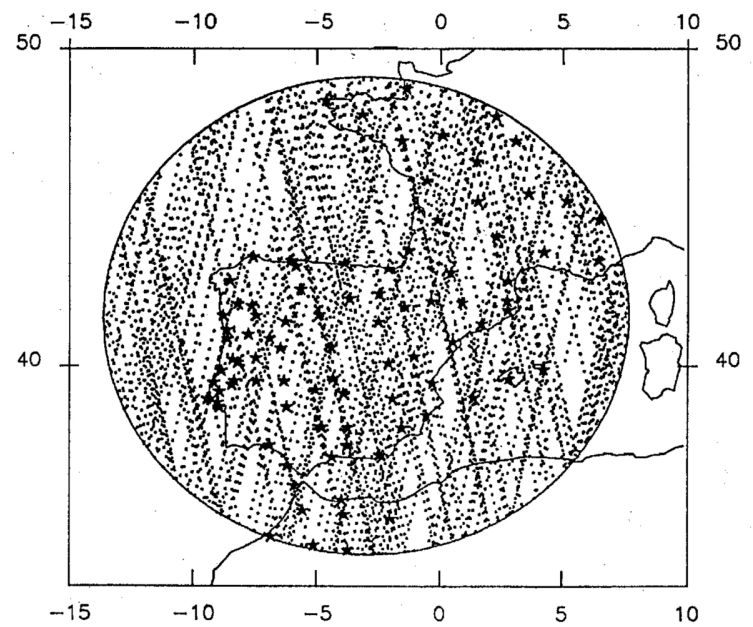

Fig. 1. Distribution of Magsat satellite data (dots) and ground data (stars). The $8^{\circ}$ spherical cap boundary is shown. Satellite data were taken between 350 and $500 \mathrm{~km}$ of altitude. 
(mainly from repeat stations), and consisted of 90 three-component values. The distributions of both data sets are shown in Fig. 1.

\section{Fixed Field Spherical Cap Harmonic Analysis}

The satellite data were processed with the aim of obtaining values due to the fixed field only: Firstly a global model, GSFC(12/83) (LANGEL and ESTES, 1985), was subtracted. GSFC(12/83) is probably the most appropriate model to evaluate the magnetic field at satellite altitude within the Magsat satellite lifetime. This model was used to remove primary and induced (degree 1) magnetospheric fields. As suggested by LANGEL and ESTES (1985, p. 2500), the external terms were derived as linear functions of $D s t$ (within the $\pm 20 \mathrm{nT}$ range of the model; the passes which crossed the spherical cap during intervals with higher Dst values were discarded); while the effect of induced fields, an internal source, was included by modifying the axial dipole term of the main field model with a term proportional to the axial term in the external field model.

Secondly second-degree polynomials were fitted to the residual values obtained for each component along each pass, in the segment between the parallels $14^{\circ} \mathrm{N}$ and $54^{\circ} \mathrm{N}$; and then, the corresponding polynomials were subtracted from the values. This was done because, according to other authors (e.g., MAYHEW, 1979; TAYLOR and FRAWLEY, 1987), selection and handling of data that are characterized by only core and crustal field contributions are rather critical. The removal of a polynomial (functions of distance along the satellite pass, or, more simply, functions of the latitude) from the satellite data should minimize the effects that result from inadequate modelling of external fields. This is shown in Fig. 2, where we compare the residuals of three different passes along similar paths, and how the removal of quadratic fits to the residual data improves pass to pass consistency. Obviously, prior to the polynomial removal, the data were taken at a common epoch (with GSFC(12/83), which also provides SV during the satellite lifetime). A small part of the signal removed could include some of the very long-wavelength crustal content (see Fig. 3), which "gives rise to a more internally consistent data set but does not establish the true anomaly zero level" (MAYHEW et al., 1985), but in our opinion this is the price to pay if we want to remove any external or induced contribution. After the polynomial removal to the residuals, we added back the main field content of GSFC(12/83) in order to have the absolute values at that specific epoch (i.e., around 1980) clean of any external field.

All the (satellite and ground) data were updated to 1990 using the $45^{\circ} \mathrm{SCH}$ SV model. Finally, as suggested by HAINES (1985a) as a method of improving convergence, before applying SCHA, the IGRF 1985 evaluated at 1990 was removed and only the residuals were analyzed. Nevertheless, perhaps the main reason for subtracting a global model is that in this way we remove the very long wavelength content of the analyzed field, which would be very difficult to fit with SCHA over a small cap-like region (TORTA et al., 1992).

Several analyses were made, slightly varying the value of $K$ (Table 2 ), modelling ground data independently, satellite data independently and finally satellite and ground data jointly. For a $8^{\circ}$ cap halfangle and $K=9$, the SCH model represents features with a minimum wavelength of $376 \mathrm{~km}$ (although,

Table 2. Standard errors, in $\mathrm{nT}$, for $\mathrm{SCH}$ models derived from satellite and ground residual data for a $8^{\circ}$ cap with different values of $K$.

\begin{tabular}{ccccc}
\hline$K$ & Only ground data & Only satellite data & Satellite + Ground & $\begin{array}{c}\text { Decimated satellite } \\
\text { + Ground }\end{array}$ \\
\hline 6 & 35.36 & 5.68 & 10.71 & 22.93 \\
7 & 34.72 & 4.55 & 10.30 & 22.18 \\
8 & 34.22 & 3.84 & 9.37 & 21.42 \\
9 & 33.97 & 3.78 & 8.83 & 20.27 \\
\hline
\end{tabular}




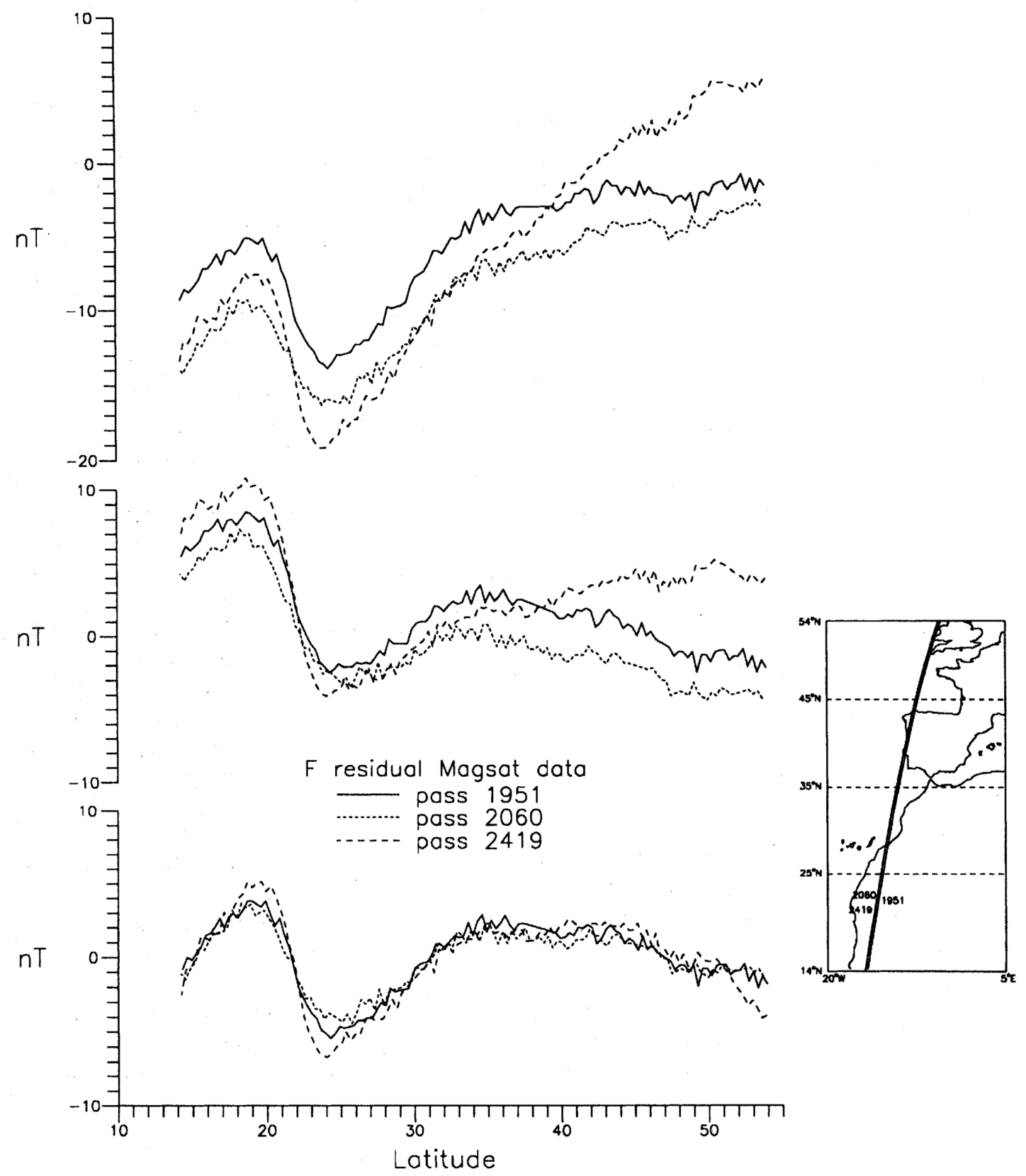

Fig. 2. Satellite scalar residual profiles with locations shown on the joined map. Top: Residuals after subtracting GSFC(12/83), including main field and SV. Middle: Residuals after subtracting GSFC(12/83), including main field, SV, and the Dst corrections for the primary and induced magnetospheric fields. The differences among the coincident passes indicate that the Dst corrections are not sufficient. Bottom: The same as Middle, but after subtracting second-degree polynomials fitted to the residual values in each pass (see Fig. 3). Similar behaviour is found for any of the field components and for any other group of coincident passes. 


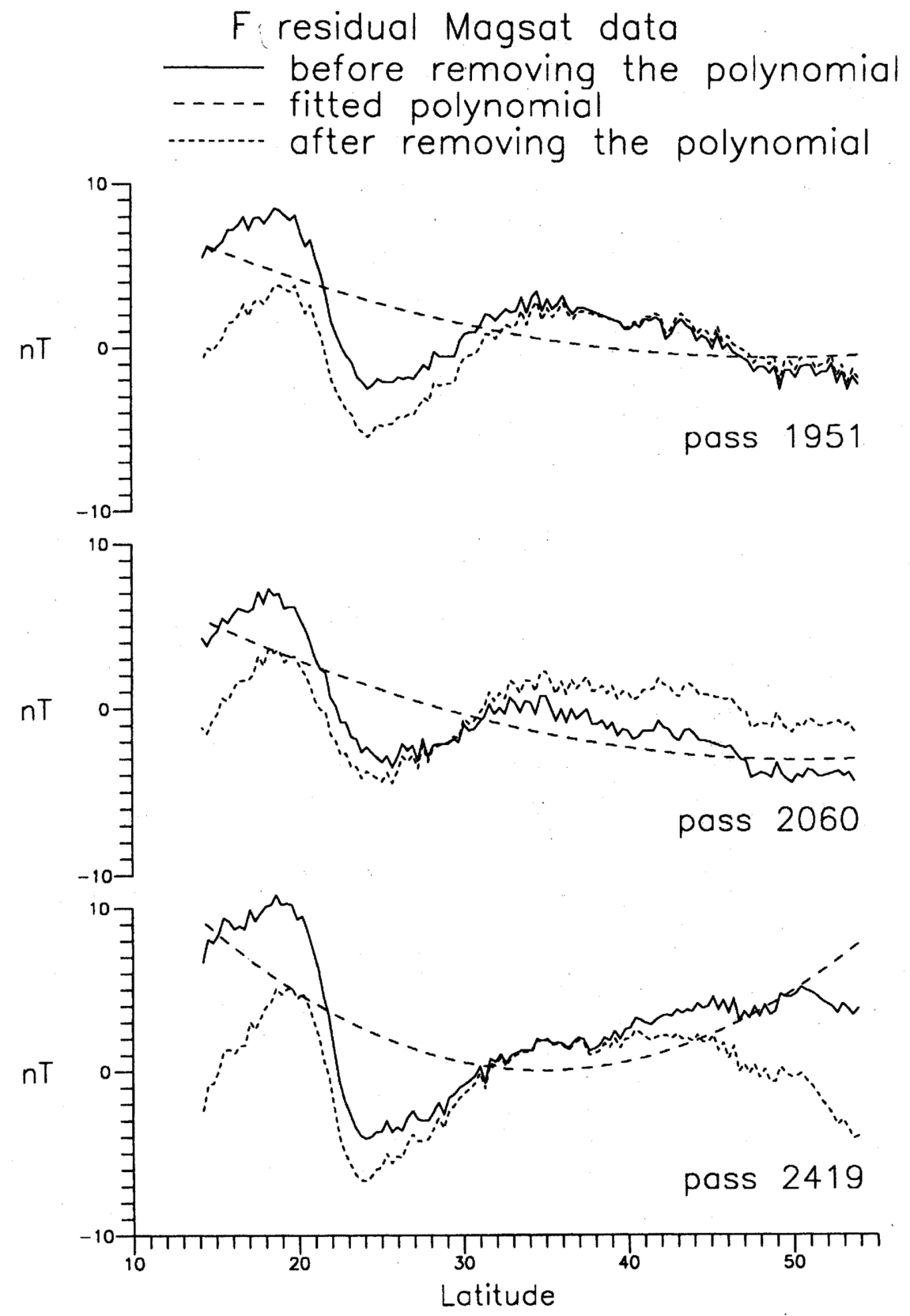

Fig. 3. Differences between the scalar residual profiles of middle and bottom of Fig. 2 (before and after removing the polynomials). The fitted polynomials are also shown.

if the model coefficient associated with this maximum degree is not significant, this minimum wavelength can increase). Such a model seems to be reasonable because its characteristic shortest wavelength is more than twice the mean distance between the ground data, with higher $K$ values we would have wavelengths around the mean distance, which are indistinguishable from those in the "Nyquist band" (BLACKMAN and TUKEY, 1958, p. 31). At this point we proceeded to plot, for the Earth's surface, the residual maps obtained 

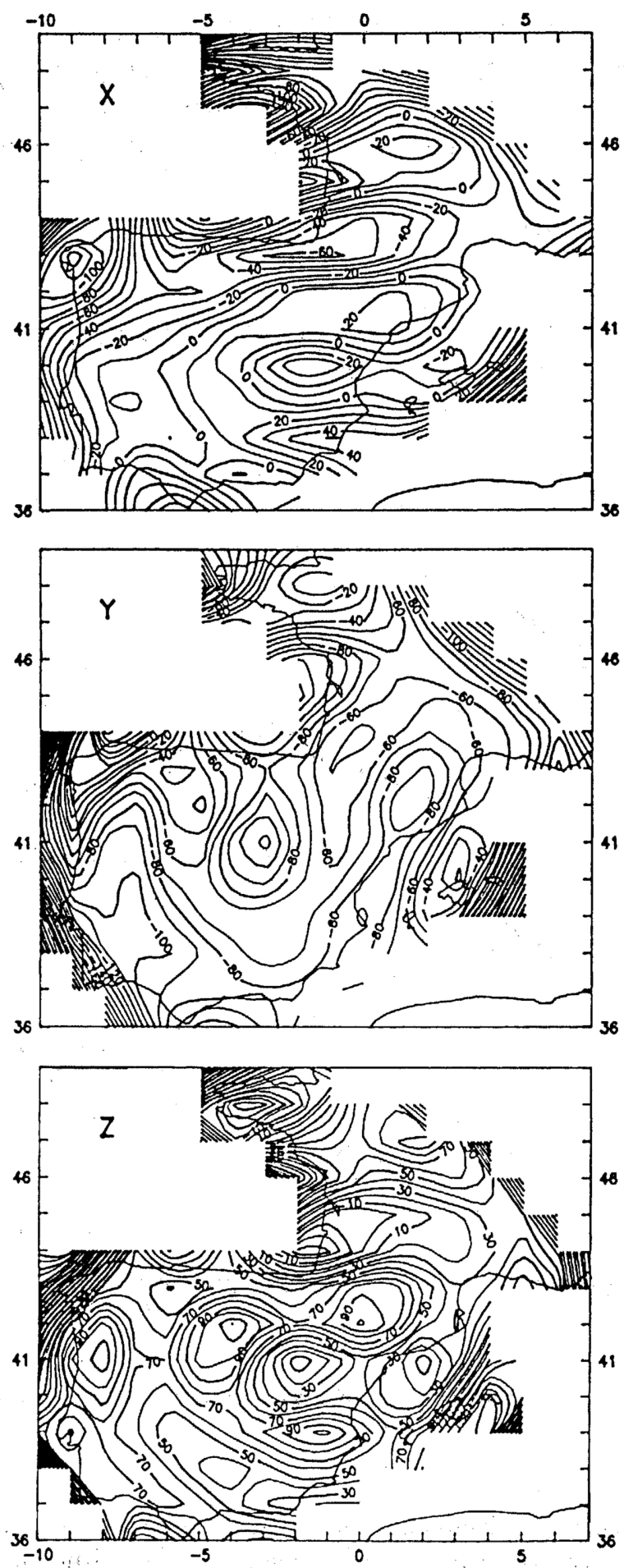

Fig. 4. Maps of the north $(X)$, east $(Y)$ and vertical $(Z)$ components for 1990.0 at Earth's surface obtained from SCHA $(K=9)$ applied to 5615 satellite and 90 ground 3 -component residual data. The contour interval is $10 \mathrm{nT}$. Contours outside the ground data area are not included because, essentially, they show only satellite data continuation errors. 

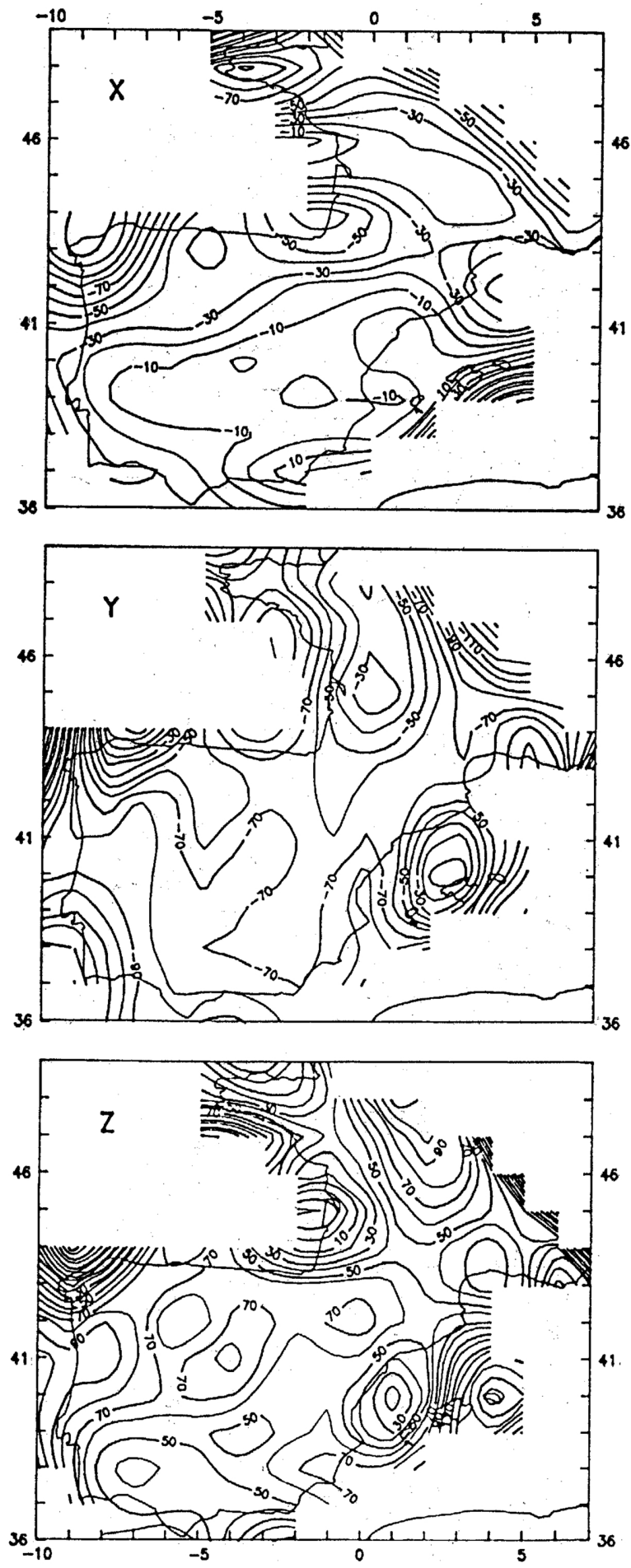

Fig. 5. Maps of the $X, Y$ and $Z$ components for 1990.0 at Earth's surface obtained from SCHA $(K=9)$ applied to 201 satellite and 90 ground 3-component residual data. The contour interval is $10 \mathrm{nT}$. 

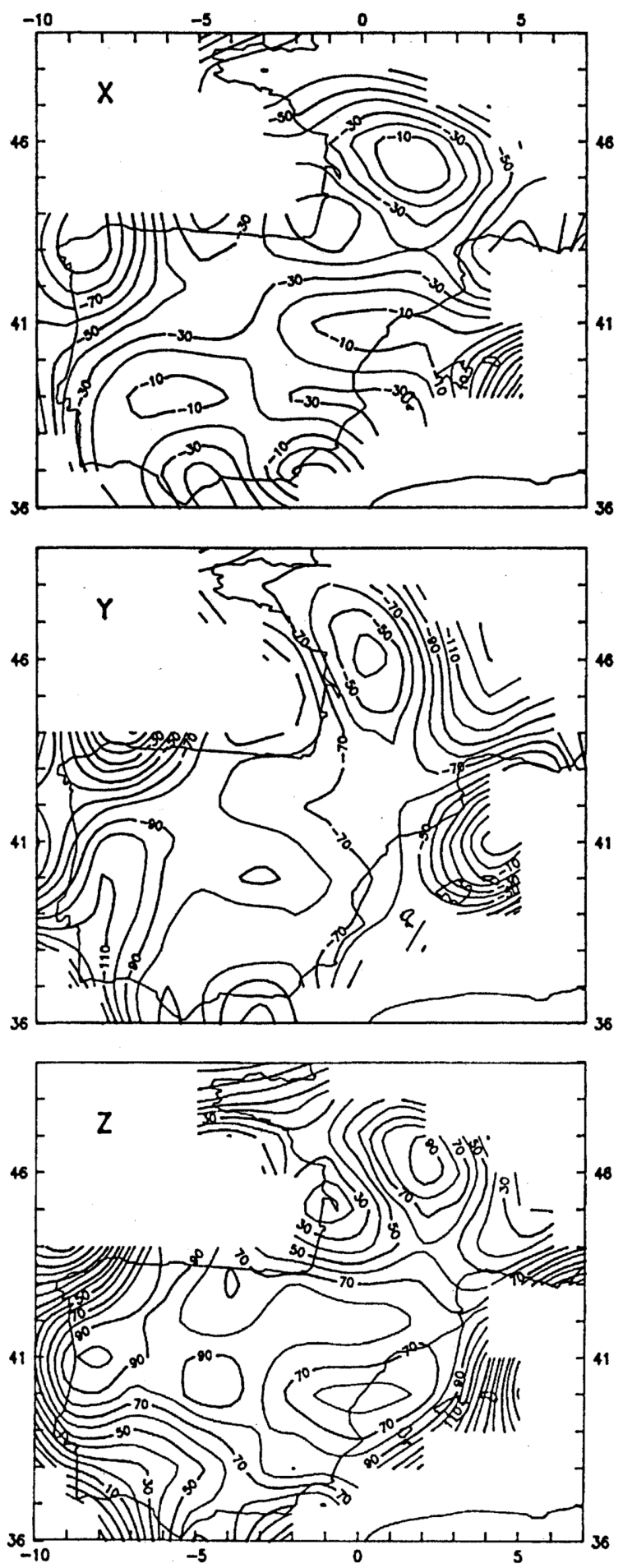

Fig. 6. Maps of the $X, Y$ and $Z$ components for 1990.0 at the Earth's surface obtained by TOSCA applied to selected satellite and ground residual data. The contour interval is $10 \mathrm{nT}$. 
from the model based on both satellite and ground data (Fig. 4). The first impression was that the residual field obtained seemed to alternate its maxima and minima along the satellite tracks, indicating that the satellite data had too much influence on the model compared to the ground data. The error in the $n$-th harmonic term at the satellite distance $r$ is magnified by a factor $(r / a)^{n+2}$ in a downward continuation to the surface of radius $a$ (HAINES and NEWITT, 1986). This fact implies that the downward continuation of satellite measurements to the Earth's surface for high degrees is not as accurate as it is for low degrees. To overcome this problem it was thought sensible to decimate the satellite data in some way, and give more weight to the ground data, because of the need for accuracy near the ground. On the other hand, it was important to include the satellite data in the analysis, because they provide good coverage over the region and furnish information on the radial variation of the field. The satellite data decimation was carried out by dividing the region inside the spherical cap into cells of approximately $1^{\circ}$ by $1^{\circ}$ (at the Equator) equal area (for details see DE SANTIS et al. (1989)). The satellite data were randomly selected by taking one data point from all those that fell inside a cell. For a cap of half-angle $8^{\circ}$ this procedure gives 201 cells. These selected satellite data, merged with the 90 measurements at the Earth's surface were analyzed again, giving the scatters shown in Table 2 (different random selections of satellite data gave standard errors similar to those reported in Table 2). The new residual maps obtained with the model with $K=9$ are also shown (Fig. 5).

These maps are more realistic with respect to the field that is actually being observed, mainly for the features of regions with ground data available. Some artificial oscillations appear in areas without ground data, especially at the border of the cap. This problem is encountered whenever the data are not well distributed over the region of interest (e.g. DE SANTIS et al., 1990). A way to overcome this drawback was

Table 3. Statistically significant coefficients, in units of nT, of the translated origin spherical cap harmonic fit $(K=9)$ to decimated satellite and ground data updated to 1990.0, after subtraction of the IGRF 1985 extrapolated to 1990.

\begin{tabular}{rrrrr}
\hline$k$ & $m$ & $n_{k}(m)$ & $G_{k}{ }^{m}$ & $H_{k}{ }^{m}$ \\
\hline 0 & 0 & .0000 & -47 & \\
1 & 0 & 4.0837 & -7 & \\
1 & 1 & 3.1196 & -27 & 152 \\
2 & 1 & 6.8354 & 13 & -206 \\
2 & 2 & 5.4928 & 6 & 55 \\
3 & 1 & 9.7121 & -4 & 189 \\
3 & 2 & 9.3733 & 0 & -91 \\
4 & 1 & 12.9083 & 0 & -87 \\
4 & 2 & 12.3720 & 0 & 96 \\
4 & 4 & 9.9589 & -8 & -46 \\
5 & 1 & 15.8215 & 0 & 20 \\
5 & 2 & 15.6154 & 0 & -59 \\
5 & 3 & 14.9180 & 5 & 0 \\
5 & 4 & 14.1778 & 0 & 55 \\
5 & 5 & 12.1334 & 0 & -16 \\
6 & 2 & 18.5830 & 0 & 19 \\
6 & 4 & 17.3910 & 0 & -39 \\
6 & 6 & 14.2861 & 7 & 0 \\
7 & 3 & 21.2461 & -8 & 0 \\
7 & 4 & 20.7588 & 0 & 9 \\
7 & 5 & 19.8121 & 0 & 17 \\
7 & 7 & 16.4228 & -5 & 0 \\
8 & 3 & 24.4170 & 4 & 0 \\
8 & 5 & 23.2437 & 0 & -8 \\
8 & 7 & 21.0663 & 0 & 15 \\
9 & 7 & 24.5451 & 0 & -7 \\
\hline
\end{tabular}


proposed by DE SANTIS (1991) who found that moving the origin of the coordinate system away from the Earth's center and toward the surface, and then applying SCHA in the new system allows smoothing of the peripheral features (the change of coordinates means that the Earth's surface is no longer equidistant from the origin so the minimum wavelength described by the new model is smaller at the centre of the region than at the edge). This filtering at the borders decreases the spurious edge oscillations of the model. Laplace's equation is not violated and the same algorithms (HAINES, 1988) can be applied in the new coordinate system.

This method, Translated Origin SCHA (TOSCA), was applied in a similar manner to that of DE SANTIS (1991) who modelled data over Italy: we chose a translated cap of half-angle $30^{\circ}$ (with the same pole as the $8^{\circ}$ cap), which involves, for the original cap used here, a vertical translation of the origin of $4773.4 \mathrm{~km}$. Figure 6 shows the residual maps obtained with this last model: the maps show more realistic behaviour, the price to pay is a small increase in the overall standard error $(\mathrm{SE}=20.99 \mathrm{nT})$, together with an increase of the minimum wavelength in comparison with the corresponding SCH model, because the harmonics with highest $n_{k}$ were not statistically significant in this analysis. The coefficients of this model are listed in Table 3. This model-plus IGRF-represents the contribution of the integration constant term of Eq. (7). The SCH coefficients of Table 3 correspond to a SCH expansion like (1) with, of course, no temporal dependence (therefore, they are simply $G_{k, 0}{ }^{m}$ and $H_{k, 0}{ }^{m}$ of Eq. (1)).

\section{Charts of the Geomagnetic Reference Field for Spain}

After the considerations described above it was a clear decision about what kind of models to adopt for the reference field model over Spain and neighbouring areas, with particular attention in SV modelling to model also the Canary Islands. The SV model here applied is the one which used SCHA $(K=4, Q=$ 2; $n_{\max }=8.14 ; 15$ coefficients significant at $F=4$ ) over a $45^{\circ}$ cap-like region centered at $34^{\circ} \mathrm{N}, 7^{\circ} \mathrm{W}$, nevertheless it has to be considered valid only over the cap area that extends for the first $16^{\circ}$, i.e., the area that included the data sets used for the analysis. The time validity spans the time interval of data, i.e., 1970-

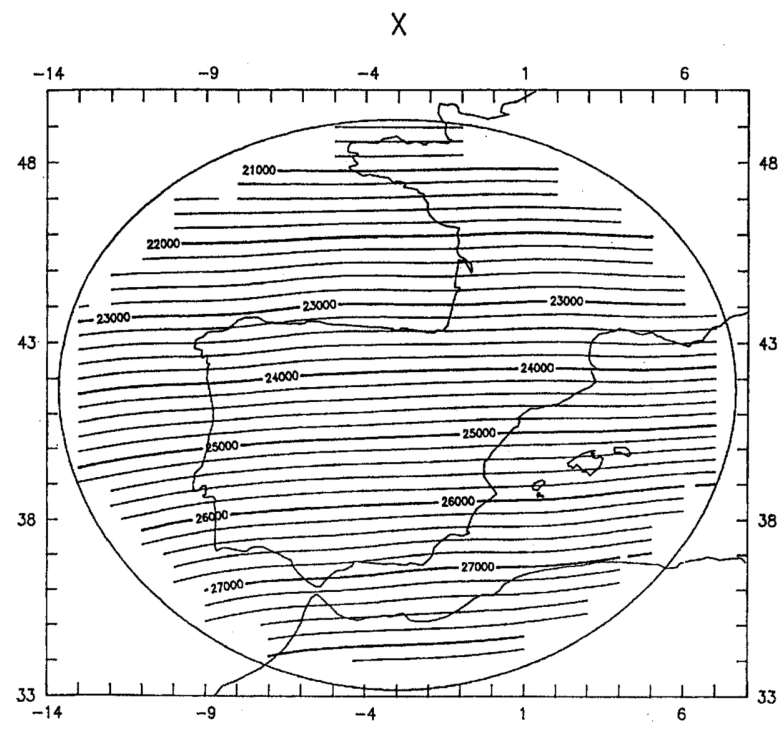

(a)

Fig. 7. Geomagnetic field elements at the Earth's surface, in nT, from the Spanish Reference Field model 1990: a) north component $(X)$, b) east component $(Y)$, c) vertical component $(Z)$. 
1989.5 , however it is our opinion that it can be extrapolated to some years beyond. This SV model has been integrated to provide main field differences from the epoch 1990.0. The integration constant is the field in 1990.

For the fixed field model, the distribution of available ground data, concentrated mainly over the European mainland, forced us to adopt the one which used TOSCA and was based on a cap-like region

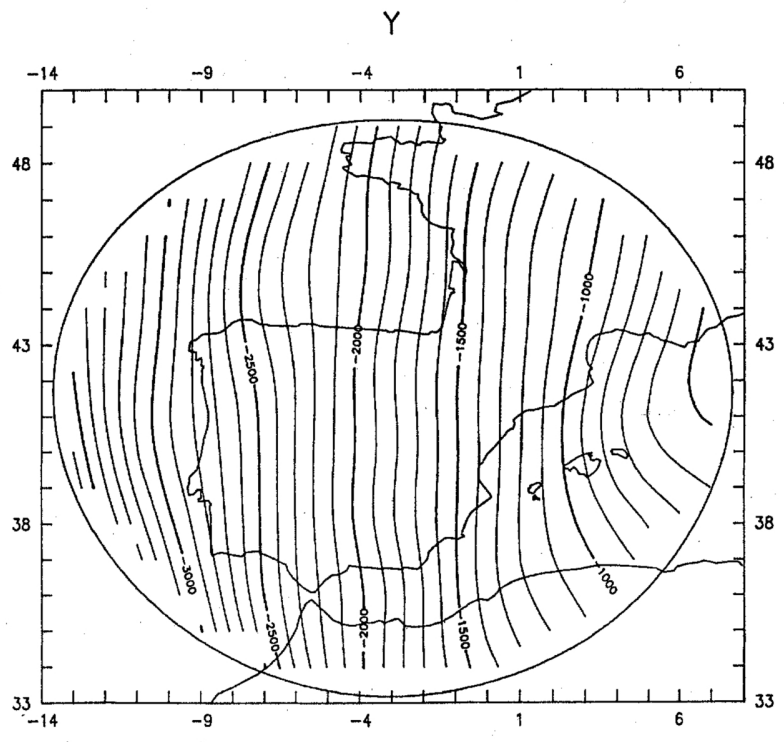

(b)

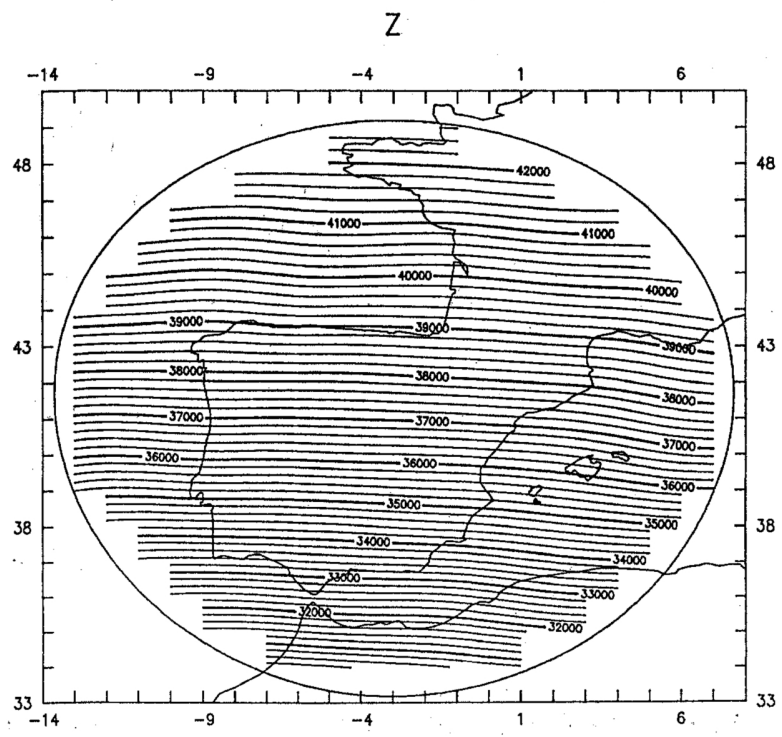

(c)

Fig. 7. (continued). 


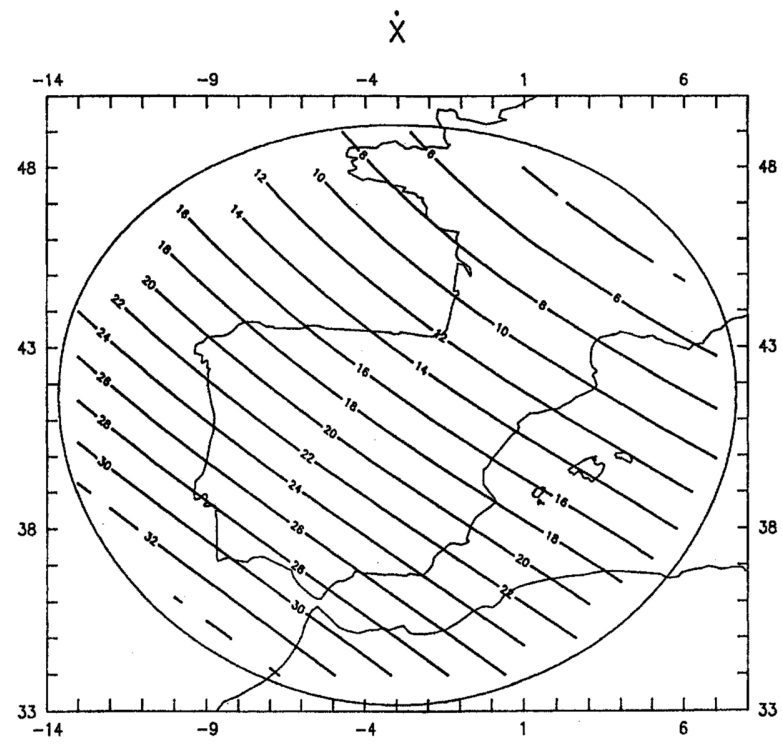

(a)

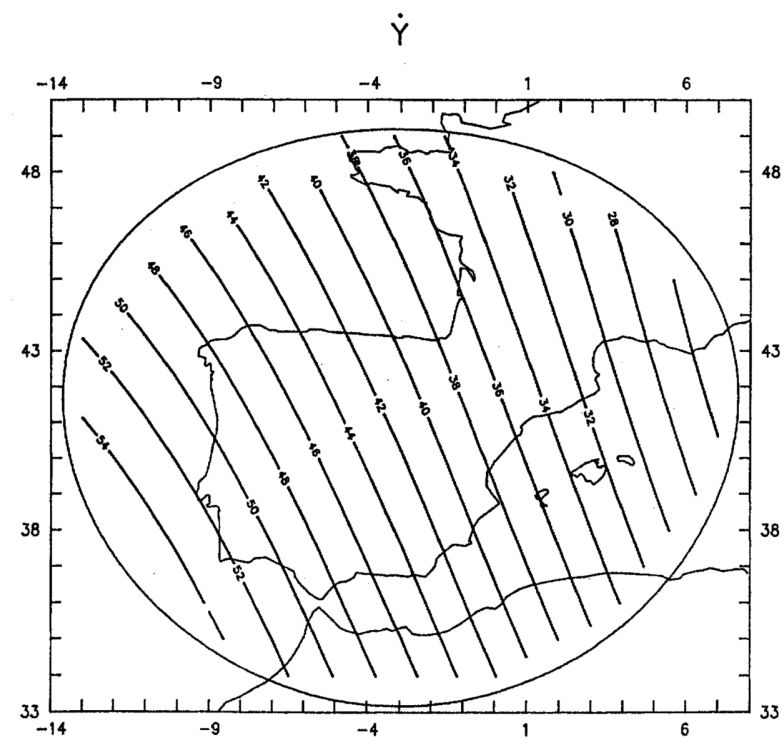

(b)

Fig. 8. Isoporic maps, in $\mathrm{nT} /$ year, for a) north component $(\dot{X}), \mathrm{b})$ east component $(\dot{Y}), \mathrm{c})$ vertical component $(\dot{Z})$, from the SCH SV model for 1990.

of $8^{\circ}$, centered at $41^{\circ} \mathrm{N}, 3^{\circ} \mathrm{W}$. The TOSCA model was derived for a $30^{\circ}$ translated origin cap, using $K=$ $9\left(\lambda_{\min }=409 \mathrm{~km} ; 31\right.$ coefficients significant at $F$-level $\left.=4\right)$. Final maps of the geomagnetic components $X, Y$ and $Z$ (after the addition of IGRF 1985, evaluated at 1990) are shown in Fig. 7; their secular variations are shown in Fig. 8. Other magnetic elements are shown by TORTA (1991). These charts represent our proposal for the Spanish Geomagnetic Reference Field at 1990 with the associated SV. 
$\dot{z}$

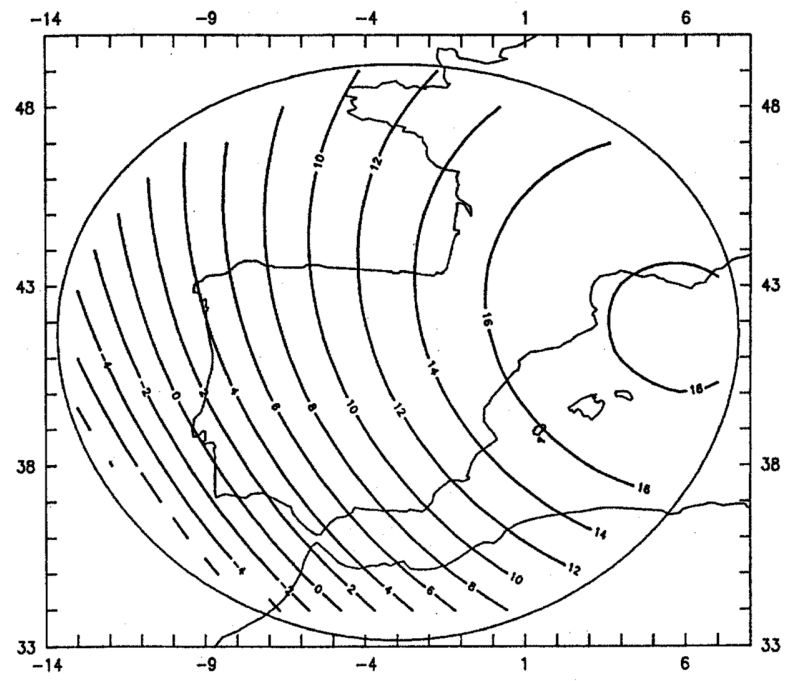

(c)

Fig. 8. (continued).

\section{Discussion}

The problem of the application of SCHA to a combination of satellite and ground data has been investigated in order to determine a reference model for the geomagnetic field at 1990 as observed in the Iberian Peninsula. It is possible to generalize some important points that emerged during the work. Removing the polynomial trend along each pass from the satellite residuals improves the fit to the ground data. Consequently, the more realistic features included in the satellite measurements are maintained when they are extrapolated to the ground. However, in applying SCHA with a non-uniform data distribution, as in this case, the extrapolation of satellite data to the Earth's surface without ground data as reference points produces huge oscillations of the modelled field components, even when some F-level criterion on the statistical significance of the model coefficients is applied. A way to solve this problem has been the use of a recent technique derived from the application of SCHA using a translated origin; the wavelengths change with the distance from the pole of the cap, and there is an effect of increasing smoothing towards the borders, where the model is most sensitive to the lack of ground data and, therefore, the downward continuation of satellite data becomes more accurate.

The authors wish to thank D. J. Kerridge for reviewing the manuscript. The paper was improved after the remarks made by D. R. Barraclough and an anonymous referee. This work is part of the research project No. PB870390 supported by the D.G.I.C.Y.T., Ministerio de Educación y Ciencia, Spain.

\section{REFERENCES}

Barraclough, D. R., IGRF: the fourth generation, Phys. Earth Planet. Inter., 48, 279-292, 1987.

Blackman, R. B. and J. W. TukEY, The Measurement of Power Spectra from the Point of View of Communications Engineering, 190 pp., Dover Publications, New York, 1958.

Bullard, E. C., The removal of trend from magnetic surveys, Earth Planet. Sci. Lett., 2, 293-300, 1967.

DE SANTIS, A., Translated origin spherical cap harmonic analysis, Geophys. J. Int., 106, 253--263, 1991. 
DE SANtis, A., D. J. KerRidge, and D. R. BARRAclough, A Spherical Cap Harmonic Model of the Crustal Magnetic Anomaly field in Europe observed by Magsat, in Geomagnetism and Palaeomagnetism, edited by F. J. Lowes, D. W. Collinson, J. H. Parry, S. K. Runcorn, D. C. Tozer, and A. Soward, 370 pp., Kluwer, Dordrecht, 1989.

DE SANTIS, A., O. BATTELLI, and D. J. KeRRIDGE, Spherical cap harmonic analysis applied to regional field for Italy, J. Geomag. Geoelectr., 42, 1019-1036, 1990.

García, A., J. M. Torta, J. J. CURTo, and E. SAnClement, Geomagnetic secular variation over Spain 1970-1988 by means of Spherical Cap Harmonic Analysis, Phys. Earth Planet. Inter., 68, 65-75, 1991 (see also Erratum, Phys. Earth Planet. Inter., 72, 135, 1992).

Golovkov, V. P. and E. M. Dimant, Summary of the Annual Mean Values of Magnetic Elements at the World Magnetic Observatories Net, Iss. XXIII, 50 pp., Academy of Sciences of the USSR, 1990.

HAINES, G. V., Spherical cap harmonic analysis, J. Geophys. Res., 90, 2583-2591, 1985a.

HAINES, G. V., Spherical Cap Harmonic analysis of geomagnetic secular variation over Canada 1960-1983, J. Geophys. Res., 90, $12563-12574,1985 \mathrm{~b}$.

HAINES, G. V., Computer programs for spherical cap harmonic analysis of potential and general fields, Comput. Geosci., 14, 4, $413-447,1988$.

HaINES, G. V., Modelling by series expansions: a discussion, J. Geomag. Geoelectr., 42, 1037-1049, 1990.

HaINEs, G. V. and L. R. NewITt, Canadian Geomagnetic Reference Field 1985, J. Geomag. Geoelectr., 38, 895-921, 1986.

LANGEL, R. A. and R. H. Estes, The near-Earth magnetic field at 1980 determined from Magsat data, J. Geophys. Res., 90, 24952509, 1985.

MAYHEW, M. A., Inversion of satellite magnetic anomaly data, J. Geophys., 45, 119-128, 1979.

MAYHEW, M. A., B. D. Johnson, and P. J. WASILEWSKI, A review of problems and progress in studies of satellite magnetic anomalies, J. Geophys. Res., 90, 2511-2522, 1985.

NewitT, L. R. and G. V. HAINES, A Canadian Geomagnetic Reference Field for epoch 1987.5, J. Geomag. Geoelectr., 41, 249$260,1989$.

TAYLOR, P. T. and J. J. FRAWLEY, Magsat anomaly data over the Kursk region, U.S.S.R., Phys. Earth Planet. Inter., 45, 255-265, 1987.

TORTA, J. M., Modelización regional del Campo Geomagnético sobre España: campo anómalo, variación secular y campo de referencia, Ph.D. Thesis, Univ. Central, Barcelona, 1991.

TorTA, J. M., J. J. CuRTO, and A. GARCIA, Geomagnetic anomaly field of Canary islands derived from Magsat satellite, Les cahiers du Centre Européen de Géodynamique et de Séismologie, 4, 179-191, 1991.

TORTA, J. M., A. GARCIA; and A. DE SANTIS, New representation of geomagnetic secular variation over restricted regions by means of SCHA: application to the case of Spain, Phys. Earth Planet. Inter., 74, 209-217, 1992. 